\title{
Liulin silicon semiconductor spectrometers as cosmic ray monitors at the high mountain observatories Jungfraujoch and Lomnický štít
}

J. Kubancak, I. Ambrozova, R. Buetikofer, K. Kudela, R. Langer, M. Davidkova, O. Ploc and Alexandr Malusek

\section{Linköping University Post Print}

\section{Tweet}

N.B.: When citing this work, cite the original article.

Original Publication:

J. Kubancak, I. Ambrozova, R. Buetikofer, K. Kudela, R. Langer, M. Davidkova, O. Ploc and Alexandr Malusek, Liulin silicon semiconductor spectrometers as cosmic ray monitors at the high mountain observatories Jungfraujoch and Lomnický štít, 2014, Journal of Instrumentation, (9), P07018.

http://dx.doi.org/10.1088/1748-0221/9/07/P07018

Copyright: IOP Publishing: Hybrid Open Access http://www.iop.org/

Postprint available at: Linköping University Electronic Press http://urn.kb.se/resolve?urn=urn:nbn:se:liu:diva-110981 


\title{
Liulin silicon semiconductor spectrometers as cosmic ray monitors at the high mountain observatories Jungfraujoch and Lomnický štít
}

\author{
Ján Kubančák, ${ }^{\mathrm{a}, \mathrm{b}, \star}$ Iva Ambrožová, ${ }^{\mathrm{a}}$ Rolf Bütikofer, ${ }^{\mathrm{c}}$ Karel Kudela, ${ }^{\mathrm{d}}$ Ronald \\ Langer, ${ }^{\mathrm{d}}$ Marie Davídková, ${ }^{\mathrm{a}}$ Ondřej Ploc $^{\mathrm{a}}$ and Alexandr Malušek \\ ${ }^{a}$ Nuclear Physics Institute of the ASCR, Department of Radiation Dosimetry \\ Na Truhlářce 39/64, 18000 Prague, Czech Republic \\ ${ }^{b}$ Czech Technical University in Prague, Faculty of Nuclear Sciences and Physical Engineering, \\ Břehová 7, 11519 Prague, Czech Republic \\ ${ }^{c}$ Physics Institute, University of Bern and International Foundation High Altitude Research Stations \\ Jungfraujoch and Gornergrat \\ Sidlerstrasse 5, CH-3012 Bern, Switzerland \\ ${ }^{d}$ Institute of Experimental Physics, Slovak Academy of Sciences \\ Watsonova 47, 04001 Košice, Slovakia \\ ${ }^{e}$ Radiation Physics, Department of Medical and Health Sciences, Linköping University, \\ SE-581 85 Linköping, Sweden \\ E-mail: jan. kubancak@odz.ujf.cas.cz
}

ABSTRACT: Currently, most cosmic ray data are obtained by detectors on satellites, aircraft, high-altitude balloons and ground (neutron monitors). In our work, we examined whether Liulin semiconductor spectrometers (simple silicon planar diode detectors with spectrometric properties) located at high mountain observatories could contribute new information to the monitoring of cosmic rays by analyzing data from selected solar events between 2005 and 2013. The decision thresholds and detection limits of these detectors placed at Jungfraujoch (Switzerland; $3475 \mathrm{~m}$ a.s.l.; vertical cut-off rigidity $4.5 \mathrm{GV}$ ) and Lomnický štít (Slovakia; $2633 \mathrm{~m}$ a.s.l.; vertical cut-off rigidity $3.84 \mathrm{GV}$ ) high-mountain observatories were determined. The data showed that only the strongest variations of the cosmic ray flux in this period were detectable. The main limitation in the performance of these detectors is their small sensitive volume and low sensitivity of the PIN photodiode to neutrons.

KEYWORDS: Dosimetry concepts and apparatus; Analysis and statistical method; Spectrometers.

\footnotetext{
* Corresponding author.
} 


\section{Contents}

1. Introduction 1

2. Materials and methods $\quad 2$

2.1 Liulin-type planar silicon diode detectors 2

2.2 Experimental setup 3

2.2.1 Lomnický štít observatory 3

2.2.2 Jungfraujoch observatory 3

2.3 Characteristic limits 3

2.4 Time necessary to detect an event 4

2.5 Barometric correction 5

2.6 Data and covered periods 5

2.7 Statistical analysis of the data 6

$\begin{array}{ll}\text { 3. Results } & \mathbf{7}\end{array}$

3.1 Characteristic limits and time necessary to detect an event $\quad 7$

3.2 Barometric coefficient 8

$\begin{array}{lr}3.3 \text { Observed heliospheric events } & 8\end{array}$

$\begin{array}{lr}\text { 3.4 Statistical analysis } & 9\end{array}$

3.5 Spectra of energy imparted 11

4. Discussion 12

5. Conclusions $\quad 13$

\section{Introduction}

The discovery of cosmic rays (CR) has given enormous impetus to large areas of science, in particular to physics. CR are often mentioned in connection with the danger they pose for human space missions, aviation and spaceship electronics. Moreover, large changes in the geomagnetic field during geomagnetic storms caused by a solar wind shock wave may have an effect on electric power production and distribution facilities. The field evolved in various directions. For instance theoretical models predicting the dynamics of solar particle events have been developed [1-3]. Validation of these models requires a wide range of experimental data that are difficult to collect.

We distinguish two major CR components - solar (solar energetic particles; SEP) and galactic (galactic cosmic rays; GCR). Intensity of GCR depends on the solar cycle phase. SEPs are produced by solar flares and/or coronal mass ejections and depending on their time profile structure are classified as impulsive or gradual [4]. Ejected solar plasma travels through interplanetary space and temporarily deforms the interplanetary and the planetary magnetic fields [1]. As a consequence, the GCR are partially shielded and their intensity is decreased; 
strong decreases are known as Forbush decreases. Mechanisms leading to SEP acceleration are not fully understood yet. Acceleration in electric fields, stochastic wave-particle interaction, or shock acceleration mechanisms can play important roles [2]. As a consequence, contemporary theoretical models are limited [3].

Currently, there are three major ways of obtaining cosmic ray data: a) measurements in space; b) measurements at high-altitudes on board balloons and aircraft [5] as well as c) measurements on the ground.

Our paper focuses on the latter, in particular on measurements at high mountain observatories (HMO), where radiation fields are dominated by secondary particles produced by interactions of the CR with the Earth's atmosphere; more detailed information about composition and spectra of these fields can be found e.g. in [6].

Over the years, the neutron monitors (NMs) became the most common instrument to monitor CR at the ground. The NMs allow continuous CR monitoring by detecting the nucleon component of the secondary cosmic ray flux, i.e. the particle flux produced by interactions of primary cosmic ray particles with the nuclei of the atmospheric atoms and molecules [7]. A single NM can be used to assess the CR spectra hardness via measurement of the multiplicity, and the worldwide network of NMs can act as a large magnetic spectrometer in the energy range from about $500 \mathrm{MeV}$ up to $15 \mathrm{GeV}$ [8]. Nevertheless, the spectrometric properties of a single NM are limited. Unlike the NMs, semiconductor detectors can provide such information but their disadvantage is a much smaller active volume.

An example of such a semiconductor detector is the Bulgarian silicon semiconductor spectrometer Liulin [9]. For example, the Liulin placed on-board a Czech Airlines commercial jet aircraft successfully registered the solar cosmic ray event on 15 April 2001 and the following Forbush decrease [10]. Several Liulin detectors have been installed at the high-mountain observatories, for instance at Moussala (Bulgaria, 2925m), Jungfraujoch (Switzerland, 3475m) and Lomnický štít (Slovakia, $2632 \mathrm{~m}$ ) observatories [11-13].

Recent results from similar experiments focused on long-term monitoring of the environmental radiation at the ground level show that such measurements can be used to demonstrate the periodicities in the solar activity [14].

The aim of this work is to evaluate whether the Liulin detector placed at HMO can provide additional information about the CR in comparison with the NMs. The ability of the Liulin to measure changes in the cosmic ray particle flux and spectra of energy imparted to its sensitive volume is discussed based on measurements made at the HMO Jungfraujoch and Lomnický štít.

\section{Materials and methods}

\subsection{Liulin-type planar silicon diode detectors}

Liulin-type detectors are 256-channel active silicon semiconductor spectrometers of energy imparted to its sensitive volume [15]. They use the HAMAMATSU S2744-08 PIN diode with an effective area of $10 \mathrm{~mm} \times 20 \mathrm{~mm}$ and thickness of $300 \mu \mathrm{m}$ as the sensitive volume [16]. The relation between energy imparted to the sensitive volume and corresponding channel number is given in the energy range from $81.3 \mathrm{keV}$ to $20.8 \mathrm{MeV}$ as: ADC Channel No. $\times 81.3 \mathrm{keV}$. This calibration equation was obtained at the Heavy Ion Medical Accelerator (HIMAC) in Chiba, Japan [17]. Liulin's accuracy is affected by (i) the uniformity of the diode thickness and (ii) the absolute dosimetric calibration of the detector in ${ }^{137} \mathrm{Cs}$ beams. According to [17], the uniformity of the $300 \mu \mathrm{m}$ diode is better than $\pm 15 \mu \mathrm{m}$; it adds an uncertainty of $5 \%$ to the energy imparted 
by a passing charged particle. The absolute dosimetric calibration adds an uncertainty of $10 \%$ [18].

\subsection{Experimental setup}

\subsubsection{Lomnický štít observatory}

At the Lomnický štít observatory (2634 $\mathrm{m}$ a.s.l.), the Liulin is installed in the living room located under the measurement room with the 8-tube NM64 type NM, see Figure 1. Both devices are operated by the Institute of Experimental Physics of the Slovak Academy of Sciences. Detailed description of the Lomnický štít observatory can be found in [19].

a)

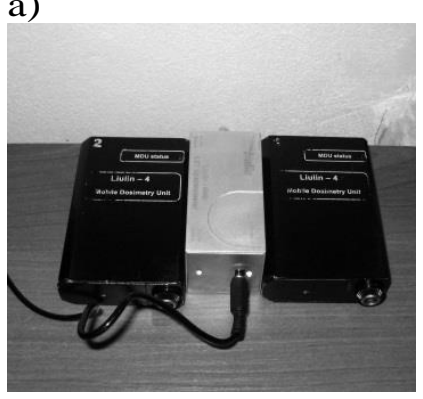

b)

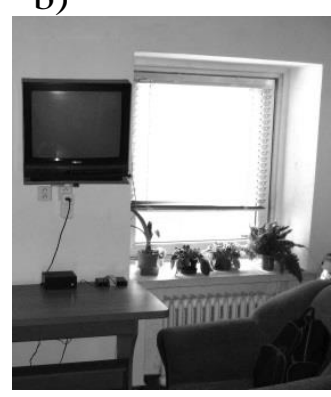

c)

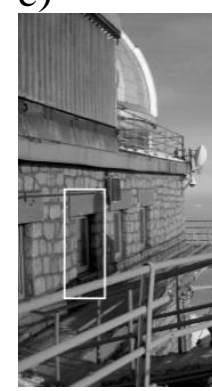

Fig. 1. a) Liulin-6I (silver one) placed at a table. b) Living room of the Lomnický štít observatory. c) The living room is located below the measurement room (its window is highlighted with the white rectangular box), which is visible as a metal building in the left upper corner.

\subsubsection{Jungfraujoch observatory}

At the Jungfraujoch, the Liulin is installed inside the NM64 NM housing at an altitude of $3475 \mathrm{~m}$ a.s.l. (see Figure 2). Both devices are operated by the Physics Institute of the University of Bern. Beside the NM64 NM, an IGY NM is also operated at the Jungfraujoch. See [20,21] for more details about the two NM types.

a)

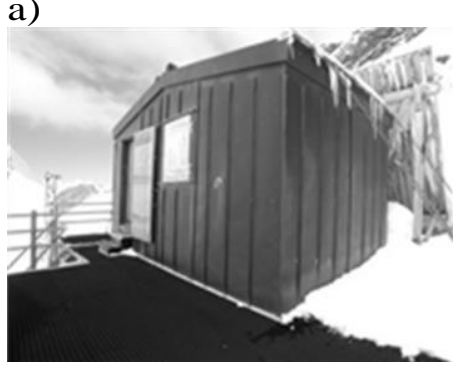

b)

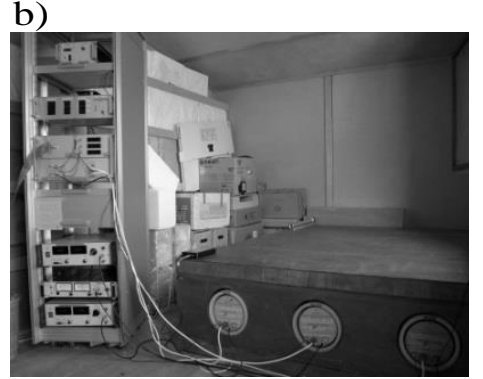

c)

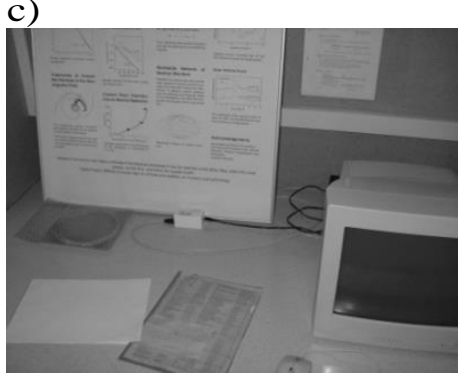

Fig. 2. a) NM-64 NM housing at the roll of the Jungfraujoch. b) Three NM counter tubes with electronic rack. c) The Liulin detector is placed at the table inside the detector housing of the NM64 NM.

\subsection{Characteristic limits}

Characteristic limits, i.e. the decision threshold and detection limit, of the Liulin detector were determined from definitions based on the ISO 11929:2010 standard [22]; the definitions were adapted to also cover measurements of the count rate decrease. Contrary to the ISO standard, which assumes normally distributed measurands, solutions presented in this work were derived for Poisson distributed measurands. Differences between the two approaches were 
small as the Poisson distribution approaches the Normal distribution for large mean values of $\lambda$ (generally for $\lambda>10$, see e.g. [23] for more details. In this work $\lambda$ was about 90).

Decision thresholds $n_{-}{ }^{*}$ and $n_{+}{ }^{*}$ for the decrease and increase, respectively, in the number of detected particles were determined from

$$
\begin{aligned}
& n_{-}{ }^{*}=K_{\mathrm{E}}\left(\alpha ; \mathrm{n}_{\mathrm{b}}\right) \\
& n_{+}{ }^{*}=K_{\mathrm{E}}\left(1-\alpha ; \mathrm{n}_{\mathrm{b}}\right)
\end{aligned}
$$

where $K_{E}\left(\alpha ; \mathrm{n}_{\mathrm{b}}\right)$ and $K_{E}\left(1-\alpha, \mathrm{n}_{\mathrm{b}}\right)$ are quantiles of the empirical distribution of the background count rate having the expectation of $n_{b}$; the quantiles were evaluated for $\alpha=0.05$.

Detection limits $n_{-}^{\#}$ and $n_{+}{ }^{\#}$ for the decrease and increase, respectively, in the number of detected particles were determined from

$$
\begin{aligned}
& K_{P}\left(1-\beta ; \mathrm{n}_{-}^{\#}\right)=n_{-}{ }^{*} \\
& K_{P}\left(\beta ; \mathrm{n}_{+}{ }^{\#}\right)=n_{+}{ }^{*}
\end{aligned}
$$

where $n_{-}{ }^{*}$ and $n_{+}{ }^{*}$ are decision thresholds defined by equations (1) and (2), respectively, and $K_{\mathrm{P}}(\beta ; \lambda)$ is the quantile function of a Poisson distribution with the mean value of $\lambda$ evaluated at $\beta$. Solutions of (3) and (4) were calculated using an iterative method, which took into account the discrete character of the Poisson distribution.

\subsection{Time necessary to detect an event}

Data acquisition time needed to detect a relative decrease $\delta$. defined as

$$
\delta_{-} \equiv \frac{\bar{N}-n_{-}^{\#}}{\bar{N}}
$$

where $\bar{N}$ is the mean number of counts detected during a 10 minute acquisition time, was derived as follows. Assume that the number of detected particles $N$ has a Poisson distribution and that $\bar{N}-n_{-}^{\#}=k \sqrt{\bar{N}}$, where $k$ is a constant that does not depend on $\bar{N}$. Then

$$
\delta_{-} \equiv \frac{\bar{N}-n_{-}^{\#}}{\bar{N}}=\frac{k \sqrt{\bar{N}}}{\bar{N}}=\frac{k}{\sqrt{\bar{N}}} .
$$

As the average number of particles detected during the acquisition time of a.10 minutes is $\bar{N}_{a \cdot 10}=a \cdot \bar{N}_{10}$, the corresponding relative decrease is

$$
\delta_{a \cdot 10}=\frac{k}{\sqrt{\bar{N}_{a \cdot 10}}}=\frac{k}{\sqrt{a} \cdot \sqrt{\bar{N}_{10}}}=\frac{\delta_{10-}}{\sqrt{a}}
$$


where $\delta_{10}$ - and $\bar{N}_{10}$ are the relative decrease and the average number of detected particles, respectively, corresponding to a 10 minute acquisition time, i.e. $\delta_{10-}=\frac{\bar{N}_{10}-n_{-}^{\#}}{\bar{N}_{10}}$. The multiple $a$ of the 10 minute acquisition time needed to detect the relative decrease $\delta$. is then given by

$$
a=\left(\frac{\delta_{10-}}{\delta_{-}}\right)^{2}
$$

Similarly for a detection of the relative count rate increase $\delta_{+}$the multiple of the 10 minute acquisition time is

$$
a=\left(\frac{\delta_{10+}}{\delta_{+}}\right)^{2}
$$

where $\delta_{10+}=\frac{n_{+}^{\#}-\bar{N}_{10}}{\bar{N}_{10}}$

The necessary times $t_{-}$and $t_{+}$for detection of the events with relative amplitudes $\delta_{-}$and $\delta_{+}$ are then given by

$$
\begin{aligned}
& t_{-}=\left(\frac{\delta_{10}}{\delta_{-}}\right)^{2} \cdot 10 \mathrm{~min} \\
& t_{+}=\left(\frac{\delta_{10}}{\delta_{+}}\right)^{2} \cdot 10 \mathrm{~min}
\end{aligned}
$$

\subsection{Barometric correction}

Atmospheric corrections for the NM data are based on theoretical and experimental investigations of meteorological phenomena that affect the passage of the particles through the atmosphere [24, 25]. The data reported in this work were corrected using [25]

$$
\mathrm{d} N=-\alpha N \mathrm{~d} p
$$

where $\alpha$ is the attenuation or barometric coefficient, $\mathrm{d} N$ is the change in the count rate $N$ and $\mathrm{d} p$ is the change of the barometric pressure. The correction was done by the authors of the NM datasets. The same method was used for the correction of the data measured with Liulin; the corresponding barometric coefficients were determined using equation (12), see [26] for more details.

\subsection{Data and covered periods}

The corrected NM data were downloaded from the real-time NM database nmdb.eu [27]. The Liulin data were downloaded from the detectors installed at the observatories. Due to the 
differences in the date of installation of both Liulin detectors, the downloaded data cover different time intervals and thereby different solar activity periods.

The Jungfraujoch measurements cover the time interval September 10 - 30, 2005 (solar minimum period) and the period from May 2012 to July 2013 (coming solar activity maximum). The Lomnický štít data cover the period from February 2011 to July 2012 (coming solar activity maximum).

During these periods, several Forbush decreases occurred and were observed by the worldwide network of NMs. However none of these events was detected by the Liulin detectors at the Jungfraujoch and Lomnický štít observatories during the overlapping period from 1 May to 6 July $2012^{1}$. Therefore, we focused on the Forbush decreases in September 2005 (for the unit placed at the Jungfraujoch observatory) and February 2011 (for the unit placed at the Lomnický štít observatory). The maximum relative amplitudes of these events measured by the NMs are presented in Table 1.

Tab. 1. Analyzed Forbush decreases. The relative NM decrease was calculated from pressure corrected NM data.

\begin{tabular}{llll}
\hline Event & Date & Observatory & Relative NM decrease \\
\hline FD 2005 & September 11, 2005 & Jungfraujoch & $14 \%$ \\
FD 2011 & February 18, 2011 & Lomnický štít & $6 \%$ \\
\hline
\end{tabular}

\subsection{Statistical analysis of the data}

Data were analyzed using the statistical computer code R [29]. Statistical tests were performed to test a) the data normality and b) possible differences in mean/median values between data obtained during normal situation and during the Forbush decrease.

Normality of the measured data was analyzed using the Shapiro-Wilk normality test: The null hypothesis $\mathrm{H}_{0}$ that the mean count rate followed the normal distribution was tested against the alternative hypothesis $\mathrm{H}_{1}$ that the data were not normally distributed; the $\mathrm{R}$ function shapiro.test $(\mathrm{x}, \ldots)$ was used. Data were visually inspected using (i) the probability density functions (PDFs) plotted using the R function density $(\mathrm{x}, \ldots)$ and (ii) the quantile-quantile (Q-Q) plots (plots of quantiles of empirical distribution against the quantiles of normal distribution normal distribution) produced using the $\mathrm{R}$ function qqnorm $(\mathrm{y}, \ldots)$.

The Wilcoxon rank sum test ( $\mathrm{R}$ function wilcox.test $(\mathrm{x}, \ldots)$ ) was used to test the null hypothesis $\mathrm{H}_{0}$ that the mean counts in periods $\mathrm{A}$ and $\mathrm{B}$ were the same against the alternative hypothesis $\mathrm{H}_{1}$ that the mean count for the period $\mathrm{B}$ was lower than that for the period A. See $[30,31]$ for more information about statistical tests. The significance level $\alpha$ was set to 0.05 in both tests.

\footnotetext{
${ }^{1}$ There occurred a Ground Level Enhancement (GLE) [26] (i.e. steep increase of the CR flux observed at the ground caused by solar CRs, see e.g. [28] Bothmer, V. and I.A. Daglis, Space Weather: Physics and Effects. 2007: Springer. ) observed by the worldwide network of neutron monitors on 17 May 2012 (GLE\#71). This GLE was only observed by high latitude neutron monitor stations and was not detected by the neutron monitors at Jungfraujoch and Lomnický štít.
} 


\section{Results}

\subsection{Characteristic limits and time necessary to detect an event}

Decision thresholds and detection limits were determined using the data obtained between 7 May 2012 and 17 May 2012. In this period, there were no space weather events observed at both observatories. The characteristic limits are presented in Table 2. Distances of the decision threshold and detection limit from the average number of detected particles relative to the average number of detected particles are presented in Table 3. Using these values we determined the time that is necessary to detect an event with a specified amplitude. Results presented in Table 4 show that at least two data acquisition cycles, i.e. 20 minutes, are needed to detect a relative increase or decrease equal to $25 \%$. An event with the relative difference of $5 \%$ from the background would have to last for more than 400 minutes to be detected. To detect an event during one data acquisition cycle, the count rate during the cycle would have to exceed the corresponding decision threshold value (see Table 2).

Tab. 2. Decision thresholds, detection limits and mean number of registered particles $\bar{N}$ for the Liulin detectors at the observatories Jungfraujoch and Lomnický štít for the acquisition time of 10 minutes. Subscripts - and + are for the decrease and increase, respectively, in the count rate.

\begin{tabular}{llllll}
\hline Observatory & $n_{-}{ }^{*}$ & $n_{+}{ }^{*}$ & $n_{-}^{\#}$ & $n_{+}{ }^{*}$ & $\bar{N}$ \\
\hline Lomnický šít & 73 & 104 & 60 & 122 & 88.3 \\
Jungfraujoch & 71 & 101 & 58 & 119 & 86.3 \\
\hline
\end{tabular}

Tab. 3. Distance of the decision threshold and detection limit (see Table 2) from the average number of detected particles relative to the average number of detected particles $\delta_{x}=\left|n_{x}-\bar{N}\right| / \bar{N}$. All values are in percent.

\begin{tabular}{lllll}
\hline Observatory & $\delta_{-}{ }^{*}$ & $\delta_{+}{ }^{*}$ & $\delta_{-}^{\#}$ & $\delta_{+}{ }^{\#}$ \\
\hline Lomnický štít & 17 & 18 & 32 & 38 \\
Jungfraujoch & 18 & 17 & 33 & 38 \\
\hline
\end{tabular}

Tab. 4. Time necessary to detect a solar heliospheric event with the Liulin detector.

\begin{tabular}{lllllllll}
\hline Time & $-5 \%$ & $5 \%$ & $-10 \%$ & $10 \%$ & $-15 \%$ & $15 \%$ & $-25 \%$ & $25 \%$ \\
\hline Lomnický štít [min] & 411 & 583 & 103 & 146 & 46 & 65 & 16 & 23 \\
Jungfraujoch [min] & 430 & 574 & 108 & 144 & 48 & 64 & 17 & 23 \\
\hline
\end{tabular}

Tab. 5. Barometric coefficients $\alpha$ for the Liulin detectors, their variance $\sigma^{2}(\alpha)$ and mean pressure $p_{0}$ to which the data are pressure corrected.

\begin{tabular}{lllll}
\hline Liulin & Period & $\alpha\left[\mathrm{mbar}^{1}\right]$ & $\sigma^{2}(\alpha)$ & $\mathrm{p}_{0}[\mathrm{mbar}]$ \\
\hline Jungfraujoch & 2013-01-31 00:00 - 2013-02-06 00:00 & $0.34 \%$ & $0.03 \%$ & 642.6 \\
Lomnický štít & 2011-11-01 00:00 - 2011-11-17 00:00 & $0.22 \%$ & $0.04 \%$ & 733.3 \\
\hline
\end{tabular}




\subsection{Barometric coefficient}

Data used for the determination of barometric coefficients for the Liulin detectors are presented in Figure 3. The resulting barometric coefficients are presented in Table 5. They are about 2-3 times lower than the barometric coefficients of both NMs (which are $0.72 \%$ / mbar). The reason is that the Liulin detector has only a small sensitivity to neutrons of the secondary cosmic rays; it detects mainly secondary charged particles and photons.

The barometric coefficients of both Liulin devices differ by 35\%. The reason is probably not only different placements and altitudes of the detectors but also their different individual sensitivities. The Liulin device at the Lomnický štít was placed inside a massive concrete building and mainly detected CR components coming from near zenith directions. The Liulin at Jungfraujoch was placed inside a wooden housing whose shielding effect was smaller.
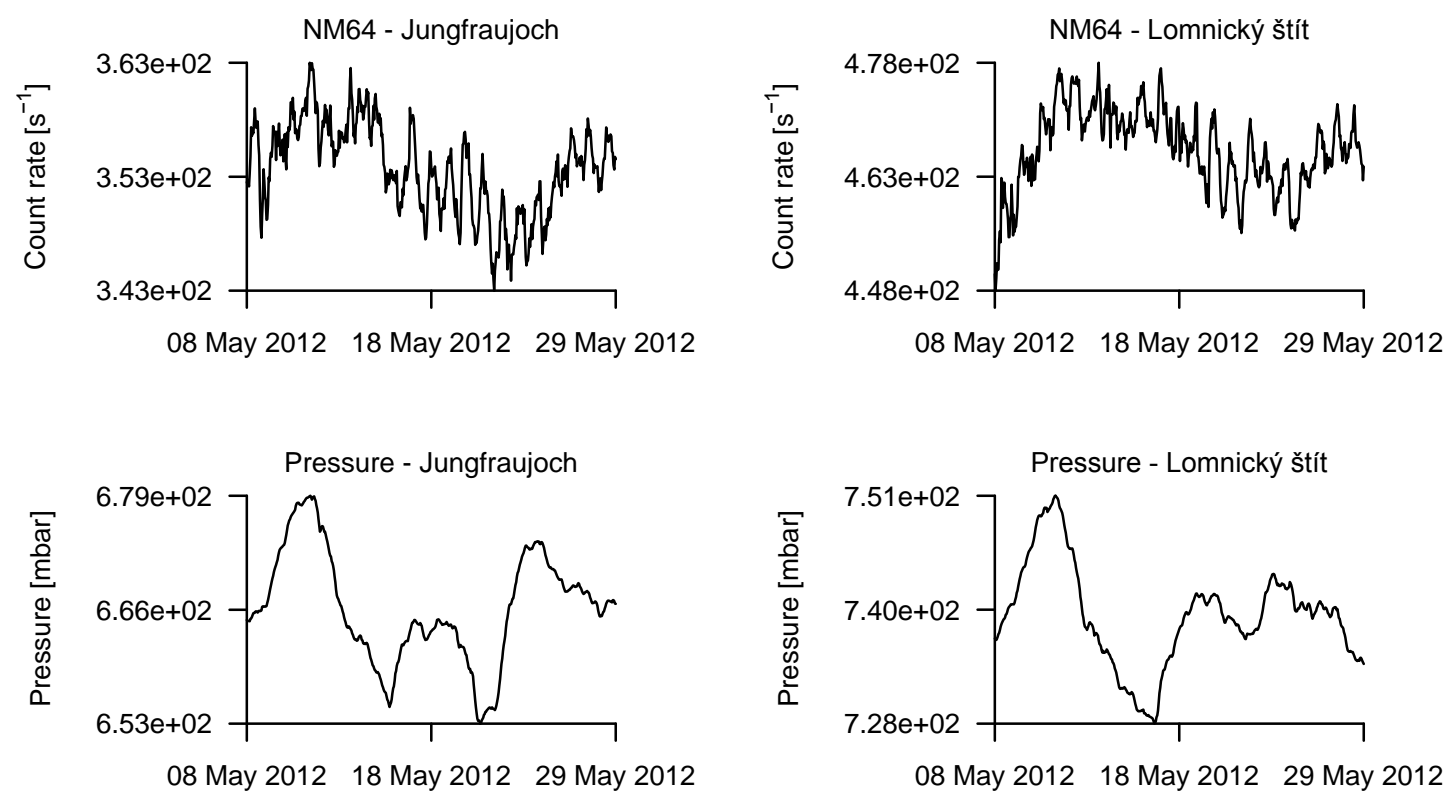

Fig. 3. Pressure corrected NM count rates and atmospheric pressure during the periods that were used for the calculation of the barometric coefficients for the Liulin detectors. The fluctuations observed in the NM data are lower than 3\% for the Jungfraujoch NM and 4\% for the Lomnický štít NM.

\subsection{Observed heliospheric events}

Data measured with the Liulin detectors and the NMs are presented in Figures 4 and 5. Figure 4 presents the data obtained at the Jungfraujoch observatory in September 2005 during a Forbush decrease with a relative amplitude of about $14 \%$ in the NM data. The decrease in the cosmic ray flux is also slightly visible in the data of the Liulin device because of its high amplitude and long duration. Figure 5 presents the data obtained with the NM at the Lomnický štít in February 2012 during a Forbush decrease. This event was not detectable by Liulin even when the data were corrected for barometric pressure, see Table 4. 

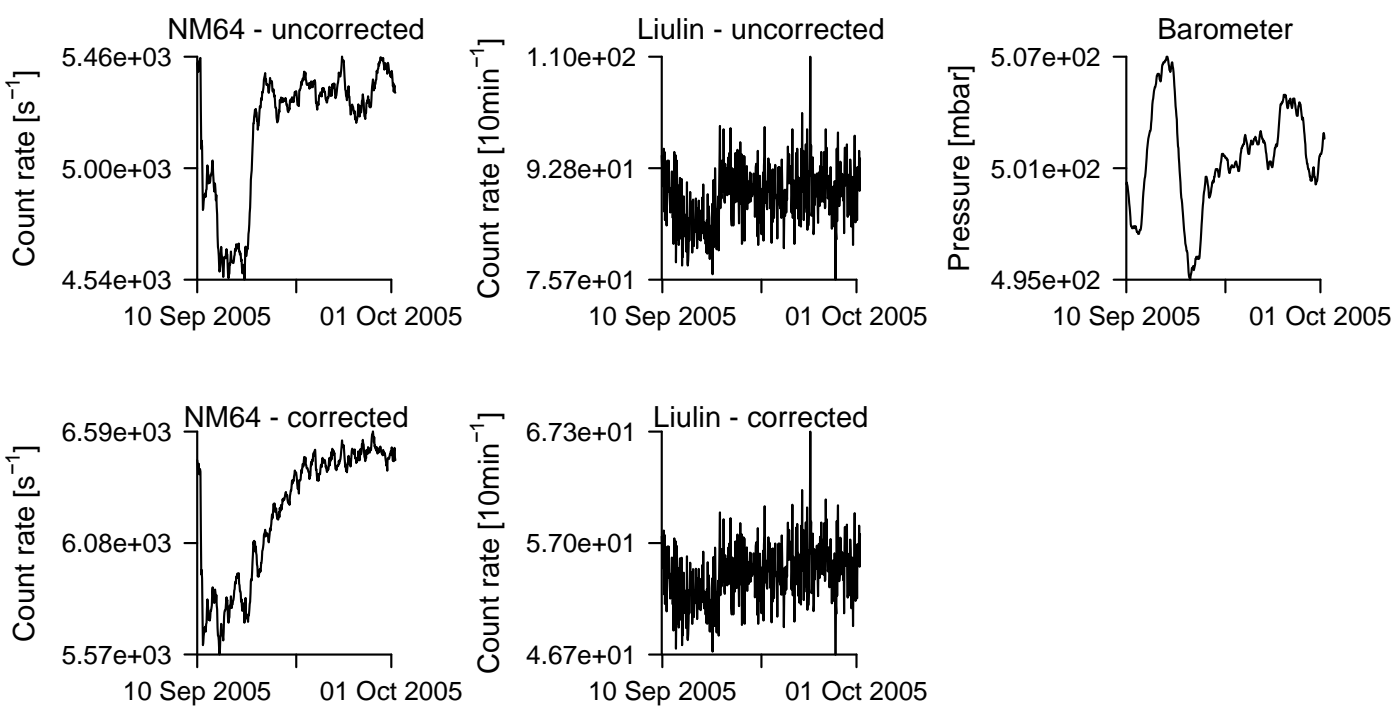

Fig. 4. The Forbush decrease observed with the Liulin detector and the NM64 NM at the Jungfraujoch observatory in September 2005. Data with and without applied barometric correction are labeled as corrected and uncorrected, respectively. The measured barometric pressure at the location of the NM64 NM is also shown.
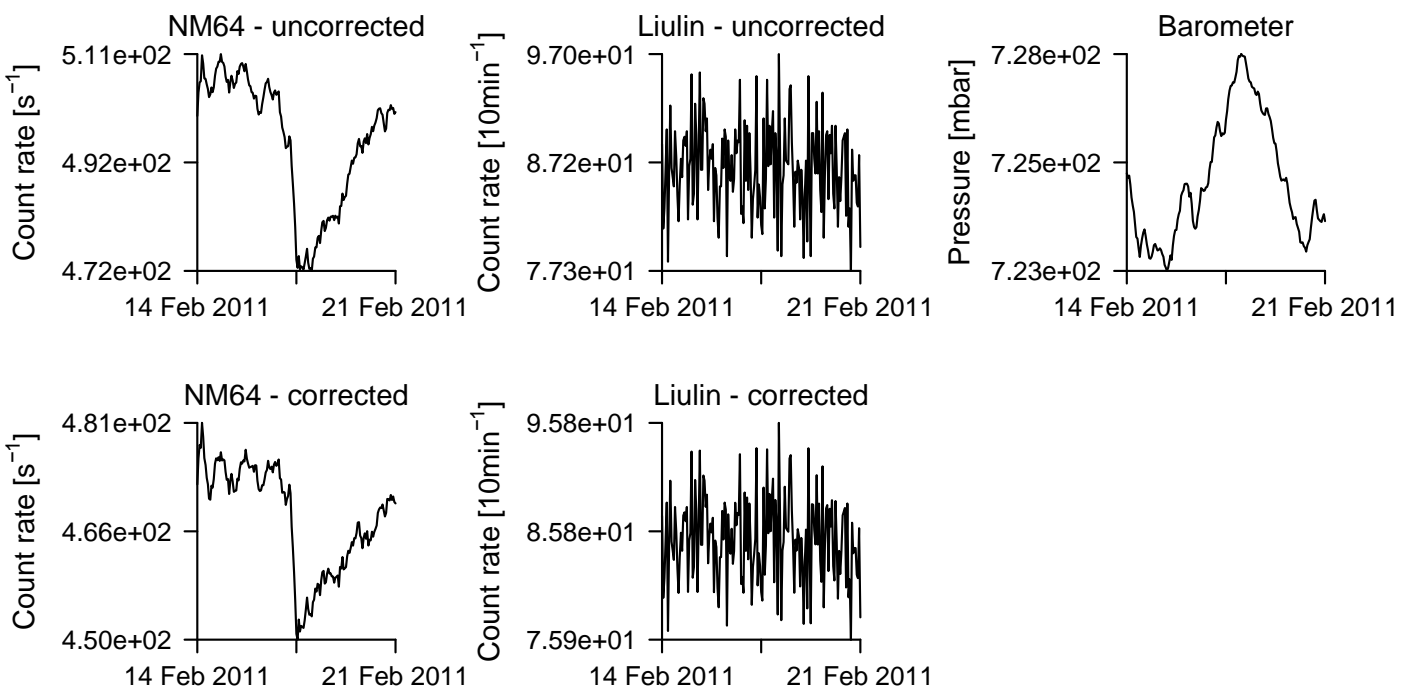

Fig. 5. The same as figure 4 for the Forbush decrease observed at the Lomnický štít observatory in February 2011.

\subsection{Statistical analysis}

The data were divided into two groups: A and B (see Table 6). The group A contains data obtained during the heliospheric event while the group B contains data obtained during times without heliospheric disturbance. 
Tab. 6. Intervals used for the statistical evaluation of measured data. Times are in local time (CEST/CET).

\begin{tabular}{lll}
\hline Event & Group A - The event is present & Group B - The event is absent \\
\hline FD 2005 & Sep 10, 2005 16:00 - Sep 20, 2005 16:00 & Sep 20, 2005 16:00 - Sep 30, 2005 16:00 \\
FD 2012 & Feb 18, 2011 00:00 - Feb 21, 2011 18:00 & Feb 14, 2011 18:00 - Feb 18, 2011 00:00 \\
\hline
\end{tabular}

Probability density plots and Q-Q plots are presented in Figures 6 and 7, respectively. The empirical PDFs differed from the theoretical normal distribution PDFs with the same mean and variance only slightly. P-values of the Shapiro-Wilk normality tests performed on groups FD 2005 A, FD 2005 B, FD 2011 A and FD 2011 B were equal to 0.004, 0, 0.09 and 0.1, respectively. Therefore, we rejected the hypothesis about the data normality for the Jungfraujoch data but not for the Lomnický štít data.

P-values of the Wilcoxon rank sum test were equal to 0 and 0.124 for the FD 2005 and FD 2011 data, respectively. Hence, we rejected the equality of mean values of the groups FD 2005 A and FD 2005 B, but we could not reject the null hypothesis $\mathrm{H}_{0}$ for the FD 2011 data.
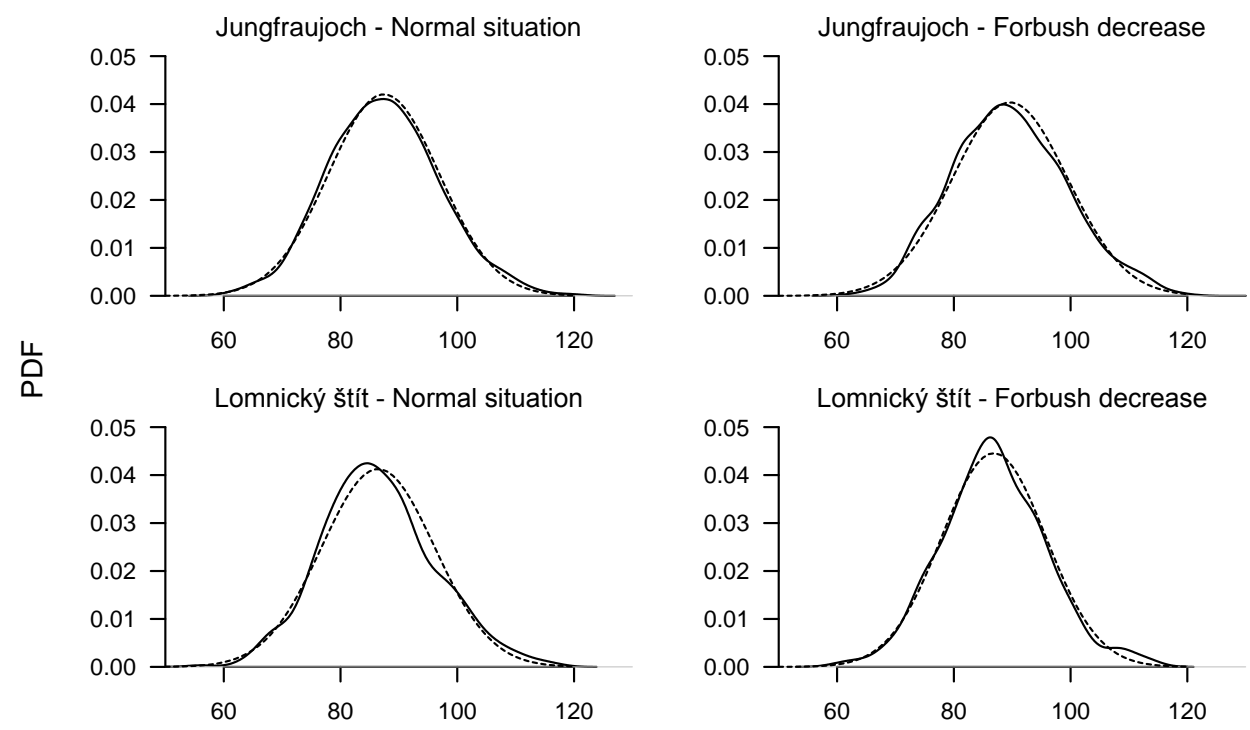

Count rate $\left[10 \mathrm{~min}^{-1}\right]$

Fig. 6. Empirical PDFs (solid lines) of the number of particles detected by Liulin during a 10 minute acquisition time and corresponding PDF of a normal distribution (dashed lines) with the mean and standard deviation taken from the data sample for periods A and B for Jungfraujoch (top) and Lomnický štít (bottom). 


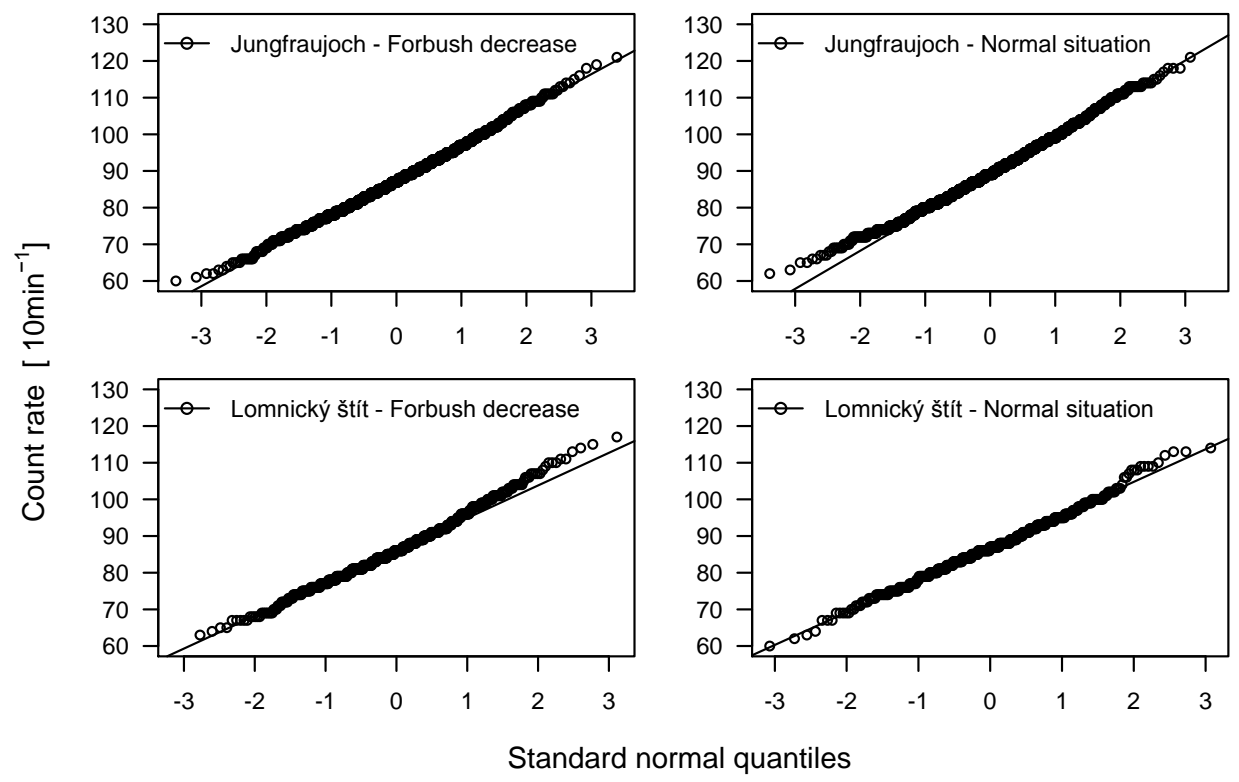

Fig. 7. Q-Q plot of the number of particles detected by Liulin during a 10 minute acquisition time for periods A (FD) and B (normal situation).

\subsection{Spectra of energy imparted}

Spectra of energy imparted to the sensitive volume of the Liulin detector obtained at both observatories are presented in Figure 8; the spectra were normalized to the total number of registered particles. Due to the high uncertainties caused by the low count numbers, we present only data from the first 64 channels, which correspond to the energy range from $81.4 \mathrm{keV}$ to 5.2 $\mathrm{MeV}$. Values of energy imparted greater than $2.2 \mathrm{MeV}$ were associated with statistical uncertainties larger than $30 \%$. Most values were lower than $1.3 \mathrm{MeV}$. These energies are typical for ionizing particles with low Linear Energy Transfer (LET) [15] like photons and electrons. The contribution of low-LET particles is demonstrated by the spectrum of energy imparted by photons emitted from a ${ }^{137} \mathrm{Cs}$ source.

Notable difference in the number of counts in the first channel between spectra from Jungfraujoch and Lomnický štít may be caused by different amount of building material surrounding the detectors as described in Section 3.2. 

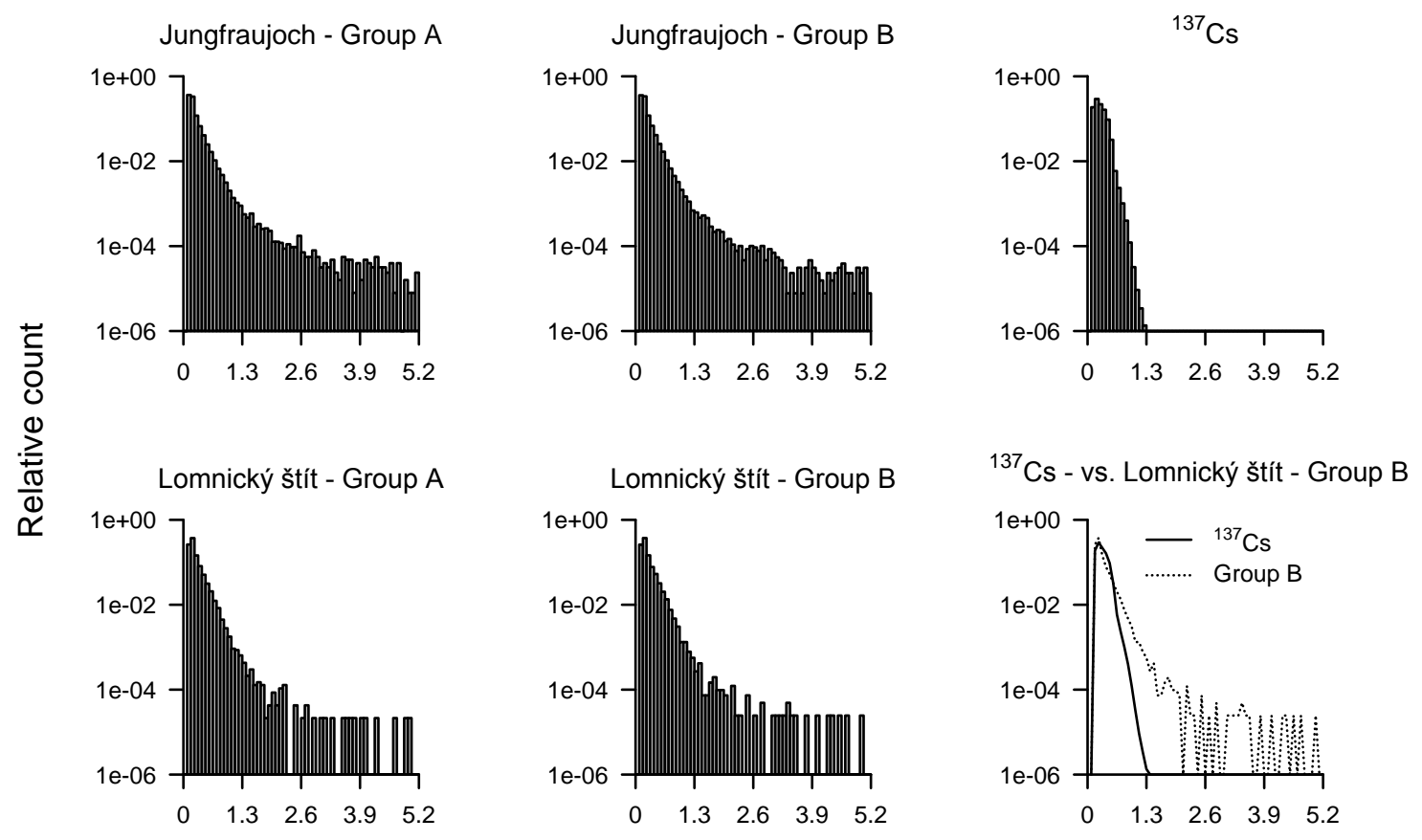

\section{Deposited Energy [MeV]}

Fig. 8. Energy imparted spectra measured with the Liulin at both observatories and in the ${ }^{137} \mathrm{Cs}$ gamma beam.

\section{Discussion}

Q-Q, kernel density plots and Shapiro-Wilk normality tests indicated a deviation from the normal distribution. The most probable causes were electronic noise and time variations in the cosmic ray flux. Detector dead time should have played a minor role [17].

Figure 8 shows that contributions to the spectrum of energy imparted were mainly from photons and charged particles with low LET. Large values of energy imparted could originate from fast neutrons; the sensitivity of the PIN diode to fast neutrons was demonstrated in Ref. [32].

The question is whether the detection efficiency of the Liulin device can be improved. If we increase the detection volume by adding more diodes, we will substantially increase the price of the device but we will not increase its thermal neutron detection capability. Sensitivity to thermal neutrons can be increased by placing a thermal neutron convertor on the diode surface. However, the problem of the convertor approach is that the HAMAMATSU S2744-08 diode has a $200 \mu \mathrm{m}$ thick protective resin layer, which effectively absorbs $\alpha$ and protons from the most common convertors like ${ }^{10} \mathrm{~B}(\mathrm{Q}=2.8 \mathrm{MeV}$ and $2.3 \mathrm{MeV}),{ }^{6} \mathrm{Li}(\mathrm{Q}=4.786 \mathrm{MeV})$ and ${ }^{3} \mathrm{He}$ $(\mathrm{Q}=764 \mathrm{keV})$ [33]. A possible solution could be the use of the diode model S2744-09, which does not have a protective resin layer.

Finally, in particular for the Lomnický štít, the detection efficiency can be increased by placing the detector outside the main observatory building. Nevertheless it is impossible at present due to the weather conditions and the static electricity induced at the observatory. 


\section{Conclusions}

Liulin detectors have relatively high decision thresholds and detection limits for cosmic ray monitoring at high-mountain observatories. The high characteristic limits are the result of the detector design, in particular the small dimensions of the sensitive volume and the low sensitivity of the PIN photodiode to neutrons.

No significant differences in the shape of energy imparted spectra collected during and in the absence of a space weather event were observed. However differences between the spectra measured at the Jungfraujoch and the Lomnický štít were apparent.

Distributions of total count numbers measured with the Liulin detectors slightly deviated from the normal distribution. Most likely, fluctuations in the cosmic ray flux during the measurement period were the main cause of the distortions.

Though Liulin's ability to detect low amplitude events at the high-mountain observatories is limited, these detectors may provide important information on rare, intense solar cosmic ray events.

\section{Acknowledgments}

The work was partially supported by grants SGS12/200/OHK4/3T/14 and by the project INGO of Ministry of Education, Youth and Sports of the Czech Republic. The neutron monitor at Lomnický štít is supported by the IEP SAS in Košice and by Slovak grant agency VEGA, project no. 2/0040/13. The operation of the neutron monitors and of the Liulin device at Jungfraujoch is supported by the Physikalisches Institut of the University of Bern and by the International Foundation High Altitude Research Stations Jungfraujoch and Gornergrat (HFSJG), CH-3012 Bern, Switzerland.

\section{References}

[1] Gopalswamy, N., R. Mewaldt, and J. Torsti, Solar eruptions and energetic particles: An introduction. Solar Eruptions and Energetic Particles, 2006. 165: p. 1-5.

[2] Aschwanden, M., et al., Theoretical Modeling for the STEREO Mission, in The STEREO Mission, C.T. Russell, Editor. 2008, Springer New York. p. 565-604.

[3] Vainio, R., et al., Dynamics of the Earth's Particle Radiation Environment. Space Science Reviews, 2009. 147(3-4): p. 187-231.

[4] Reames, D., Particle acceleration at the Sun and in the heliosphere. Space Science Reviews, 1999. 90(3-4): p. 413-491.

[5] Ploc, O., et al., Publicly available database of measurements with the silicon spectrometer Liulin onboard aircraft. Radiation Measurements, 2013. 58(0): p. 107112.

[6] Grieder, P.K.F., Cosmic rays at Earth : researcher's reference manual and data book. 1st ed. 2001, Amsterdam ; New York: Elsevier Science Ltd. xx, 1093 p.

[7] Stoker, P., L. Dorman, and J. Clem, Neutron Monitor Design Improvements, in Cosmic Rays and Earth, J.W. Bieber, et al., Editors. 2000, Springer Netherlands. p. 361-380.

[8] Nagashima, K., et al., Response and yield functions of neutron monitor, galactic cosmic-ray spectrum and its solar modulation, derived from all the available worldwide surveys. Il Nuovo Cimento C, 1989. 12(2): p. 173-209.

[9] Ploc, O., et al., Use of energy deposition spectrometer Liulin for individual monitoring of aircrew. Radiat Prot Dosimetry, 2011. 144(1-4): p. 611-4.

[10] Spurný, F., K. Kudela, and T. Dachev, Forbush decreases registered onboard aircraft. Advances in Space Research, 2005. 36(9): p. 1634-1637. 
[11] Kudela, K. and R. Langer, Cosmic ray measurements in High Tatra mountains: 19572007. Advances in Space Research, 2009. 44(10): p. 1166-1172.

[12] Mishev, A.L. and E. Hristova, Recent gamma background measurements at high mountain altitude. Journal of Environmental Radioactivity, 2012. 113: p. 77-82.

[13] Zanini, A., M. Storini, and O. Saavedra, Cosmic rays at High Mountain Observatories. Advances in Space Research, 2009. 44(10): p. 1160-1165.

[14] Babić, D., et al., Fine structure of the absorbed dose rate monitored in Zagreb, Croatia, in the period 1985-2011. Journal of Environmental Radioactivity, 2013. 118(0): p. 7579.

[15] Report 85: Fundamental quantities and units for ionizing radiation. J ICRU, 2011. 11(1): p. 1-31.

[16] Dachev, T.P., Characterization of the near Earth radiation environment by Liulin type spectrometers. Advances in Space Research, 2009. 44(12): p. 1441-1449.

[17] Uchihori, Y., et al., Analysis of the calibration results obtained with Liulin-4J spectrometer-dosimeter on protons and heavy ions. Radiation Measurements, 2002. 35(2): p. 127-134.

[18] Dachev, T., et al., Calibration results obtained with Liulin-4 type dosimeters. Space Life Sciences: Biological Research and Space Radiation, 2002. 30(4): p. 917-925.

[19] Kudela, K., et al. Neutron monitor at Lomnický stit: brief description and revised data for 12/1981-12/1999. 2000.

[20] Carmichael, H., Cosmic rays. 1964: IQSY Secretariat.

[21] Hatton, C.J. and H. Carimichael, EXPERIMENTAL INVESTIGATION OF THE NM-64 NEUTRON MONITOR. Canadian Journal of Physics, 1964. 42(12): p. 2443-2472.

[22] ISO, ISO 11929:2010 - Determination of the characteristic limits (decision threshold, detection limit and limits of the confidence interval) for measurements of ionizing radiation, 2010, International Organization for Standardization: Geneva.

[23] Ross, S.M., Introductory Statistics. 2010: Elsevier Science.

[24] Lindgren, S., On the pressure dependence of the cosmic ray intensity recorded by the standard neutron monitor. Tellus, 1962. 14(1): p. 44-48.

[25] Clem, J. and L. Dorman, Neutron Monitor Response Functions. Space Science Reviews, 2000. 93(1-2): p. 335-359.

[26] Bütikofer, R., Zum Wiederaufbau des Multiplizitätsneutronen-monitors in Bern., in Physics Institute 1988, University of Bern.

[27] NMDB. Neutron Monitor Database. 2013 [cited 2013 2013]; Available from: http://www.nmdb.eu.

[28] Bothmer, V. and I.A. Daglis, Space Weather: Physics and Effects. 2007: Springer.

[29] Jones, O., R. Maillardet, and A. Robinson, Introduction to Scientific Programming and Simulation Using R. 2012: Taylor \& Francis.

[30] Cornillon, P.A., et al., $R$ for Statistics. 2012: Taylor \& Francis.

[31] Jackson, S.L., Research methods and statistics : a critical thinking approach. 2012, Belmont, CA: Wadsworth Cengage Learning.

[32] Liyuan, Z., M. Rihua, and Z. Ren-Yuan. Neutron induced nuclear counter effect in Hamamatsu silicon PIN diodes and APDs. in Nuclear Science Symposium Conference Record (NSS/MIC), 2010 IEEE. 2010.

[33] Poenaru, D.N. and W. Greiner, Experimental Techniques in Nuclear Physics. 1997: Walter de Gruyter. 